\title{
Competencias digitales en docentes de la facultad de humanidades y ciencias sociales de la universidad adventista de Bolivia
}

\author{
Juan Wilfredo Choque Medrano \\ wilfredo.choque@uab.edu.bo \\ https://orcid.org/0000-0002-1528-227X \\ Universidad Adventista de Bolivia \\ Cochabamba - Bolivia \\ Karina Villarroel Colque \\ karina.villarroel@uab.edu.bo \\ https://orcid.org/0000-0001-6456-6562 \\ Universidad Adventista de Bolivia \\ Cochabamba - Bolivia
}

\section{RESUMEN}

El desarrollo de competencias digitales ha cobrado vital importancia por la irrupción de tecnologías y, porque en el último tiempo, la educación ha optó por la virtualidad. En este contexto, se hace necesario evaluar las competencias digitales de los docentes para conocer cómo están desarrollando procesos educativos mediados por TIC's. El estudio es descriptivo y se realizó con la participación de 29 docentes de la facultad de Humanidades y Ciencias Sociales de la Universidad Adventista de Bolivia. Se utilizó el cuestionario Tourón-Martín que evalúa cinco dimensiones: Información y alfabetización digital, Comuncación y colaboración, Creación de contenido digital, Seguridad, Resolución de problemas. Se utilizó dos escalas tipo Likert de seis puntos que valoran el conocimiento y uso del docente respecto a las competencias digitales. El resultado general muestra un nivel alcanzado de $63 \%$ en Conocimiento y $58 \%$ en Uso y una correlación alta y directamente proporcional entre ambas.

Palabras clave: competencias digitales; docencia; tecnología. 


\title{
Digital skills in teachers of the faculty of humanities and social sciences of the adventist university of Bolivia
}

\begin{abstract}
The development of digital skills has become vitally important due to the rise of new teaching technologies. In recent times, online classes was the option educators have chosen. In this context, teachers' digital skills needed an assessment in order to know how they are developing educational processes mediated by teaching technologies. This is a descriptive study. It had 29 participant teachers from the Department of Humanities and Social Sciences of the Adventist University of Bolivia. This study applied the TourónMartín questionnaire. The questionnaire assesses five dimensions: Information and Digital Literacy, Communication and Collaboration, Creation of Digital Content, Security, and Problem Solving. Two six-point Likert-type scales were used to assess the teachers' knowledge and use of digital skills. The result shows a level reached of $63 \%$ in knowledge and 58\% in use of teaching technologies. It also shows a high and proportional correlation between the two areas of assessment.
\end{abstract}

Keywords: digital skills; teaching; technology.

Artículo recibido: 05 octubre. 2021 Aceptado para publicación: 02 noviembre 2021 Correspondencia: wilfredo.choque@uab.edu.bo

Conflictos de Interés: Ninguna que declarar 


\section{INTRODUCCIÓN}

Las competencias digitales, en estos últimos tiempos se han convertido en un requisito indispensable en el desempeño docente. Debido a los recientes cambios orientados a la educación virtual, a nivel global, "las competencias digitales, antes "opcionales", se han convertido en "esenciales" (UNESCO, 2018). Esta condición, hace imperiosa la necesidad de conocer el nivel que los docentes universitarios han alcanzado en el desarrollo de su práctica educativa, en función de comunicarse de forma eficaz con los estudiantes, en las clases en línea como fuera de ellas. En en el entendido de que un docente que no tiene desarrolladas sus competencias digitales, tampoco podrá incidir en el desarrollo de dichas competencias en el estudiantado (Fernandez \& Fernández, 2016). La tecnología ha cambiado el entorno de diferentes áreas de trabajo. El uso de internet combió totalmente la capacidad de conexión e interactividad entre las personas (Lévano et al, 2019). Este hecho ha transformado los diferentes escenarios y la educación no ha quedado al margen. En concreto la educación universitaria debiera constituirse en un motor de desarrollo de competencias digitales e informacionales, rompiendo de esta manera la brecha digital intergeneracional, social y cultural (Navarro \& Barrios, 2016). Las competencias digitales son entendidas como "las habilidades, conocimientos y actitudes respecto al uso de las TIC" (Ketil, 2019, pag. 11) y orientados a facilitar el uso de las diferentes aplicaciones en entornos y dispositivos digitales con la finalidad de crear e intercambiar contenidos digitales. Por otra parte, facilitan la comunicación y colaboración dentro de una comunidad educativa, así como posibilitan la solución de problemas relacionados con el uso de tecnologías. Los docentes deben incluir, dentro sus competecias pedagógicas, las digitales. Esto se ha vuelto tan relevante que, quien no las posea, no podría desempeñarse eficazmente en la actividad docente (Lévano-Francia, Sánchez, Guillén, Tello, Herrera, Collantes, 2019).

Estudios realizados por otros investigadores, muestran los siguientes resultados. Se encontró un desarrollo mediano en las competencias mínimas docentes. Si bien los docentes tienen un nivel alto en el ámbito tecnológico, su competencia pedagógica es mas bien baja (Rangel y Peñaloza, 2013). Por su parte, Álvarez, Núñez y Rodríguez (2017) hicieron un estudio en estudiantes universitarios y encontraron que ellos muestran deficiencias en las áreas de comunicación, seguridad y solución de problemas, además que, durante su permanencia en una carrera, no alcanzaron un buen desarrollo de 
competencias digitales. Respecto a las competencias en educación virtual, la investigación de Martínez y Garcés (2020) estableció que las competencias más desarrolladas fueron: Información y alfabetización informacional, Comunicación y colaboración, además de encontrar una correlación positiva y fuerte en tres de las competencias.

El conocimiento y práctica de competencias digitales se ha tornado imprescindible en la actuación del docente del Siglo XXI. Los docentes no sólo deben conocer el uso de la tecnología, sino que, además, deben darle un uso pedagógico - didáctico. En eso justamente, radica la dificultad de incorporar TIC en educación. La competencia exige primeramente, un conocimiento, para luego transformarse en acción permanente. En tal sentido, para el presente estudio se considera como marco común de competencias digitales el expresado por (INTEF 2017; Vargas-Murillo 2019), donde se establecen las siguientes cinco.

1. Información y alfabetización informacional. Identificar, localizar, recuperar, almacenar, organizar y analizar la información digital, evaluando su finalidad y relevancia.

2. Comunicación y colaboración. Comunicar en entornos digitales, compartir recursos a través de herramientas en línea, conectar y colaborar con otros a través de herramientas digitales, interactuar y participar en comunidades y redes; conciencia intercultural.

3. Creación de contenido digital. Crear y editar contenidos nuevos, integrar y reelaborar conocimientos y contenidos previos, realizar producciones y contenidos multimedia, programación informática, aplicar derechos de propiedad intelectual y licencias de uso.

4. Seguridad. Protección personal, protección de datos, protección de la identidad digital, uso de seguridad, uso y seguro sostenible.

5. Resolución de problemas. Identificar necesidades y recursos digitales, tomar decisiones a la hora de elegir la herramienta digital apropiada, acorde a la finalidad o necesidad, resolver problemas conceptuales a través de medios digitales, resolver problemas técnicos, uso creativo de la tecnología, actualizar la competencia propia y la de otros. 
Conocer el nivel alcanzado por los docentes en relación a las competencias digitales es determinante para el ejercicio docente actual. Por ello, se consideró como objetivos del estudio a) establecer el nivel de conocimiento y uso de competencias digitales aplicadas al aprendizaje en entornos digitales de profesores universitarios, b) establecer la relación existente entre conocimiento y uso de competencias digitales.

\section{MÉTODO}

El estudio es no experimental, descriptivo pues se centró en la descripción de las características de los docentes con relación al conocimiento y uso de las competencias digitales (Veiga, Fuente, Zimmermann, 2008). También es correlacional por cuanto se pretende conocer el grado de relación entre el conocimiento y uso de competencias digitales (Hernández, 2014). El estudio utiliza un enfoque cuantitativo.

\section{POBLACIÓN}

La población estuvo constituida por 29 docentes de la Facultad de Humanidades y Ciencias Sociales de la Universidad Adventista de Bolivia. Se utilizó una muestra no probabilística por conveniencia con un error muestral de $0,05 \%$ que destaca una alta representatividad de la muestra referida al total de la población. El resultado y composición de la población resultante, de acuerdo al género, edad y experiencia de los participantes puede verse en las Tablas 1 y 2.

Tabla 1. Composición de la población por género

\begin{tabular}{|l|l|c|}
\hline \multicolumn{1}{|c|}{ Género } & $\mathbf{N}^{\circ}$ & \% \\
\hline Femenino & 16 & 55 \\
\hline Masculino & 13 & 45 \\
\hline Total & $\mathbf{2 9}$ & $\mathbf{1 0 0}$ \\
\hline
\end{tabular}

Fuente: Elaboración propia

Tabla 2. Composición de la población de acuerdo a la edad y experiencia de los participantes expresados en años

\begin{tabular}{|c|c|c|c|c|c|}
\hline Edad & $\mathbf{N}^{\circ}$ & $\mathbf{\%}$ & Experiencia & $\mathbf{N}^{\circ}$ & $\mathbf{\%}$ \\
\hline $21<30$ & 5 & 17 & $1-5$ años & 10 & 34 \\
\hline $31<40$ & 13 & 45 & $6-10$ años & 6 & 21 \\
\hline $41<50$ & 8 & 28 & $11-15$ años & 8 & 28 \\
\hline $51<60$ & 3 & 10 & $16-20$ años & 1 & 3 \\
\hline $61<70$ & 0 & 0 & $>20$ años & 4 & 14 \\
\hline Total & $\mathbf{2 9}$ & $\mathbf{1 0 0}$ & Total & $\mathbf{2 9}$ & $\mathbf{1 0 0}$ \\
\hline
\end{tabular}

Fuente: Elaboración propia. 


\section{Técnicas y herramientas de recolección de la información}

Para la recolección de información se utilizó el cuestionario Tourón - Martín (2018) que mide el conocimiento y uso de las competencias digitales. La fiabilidad del instrumento, estimada con el Alpha de Cronbach, es de 0,98. El cuestionario está compuesto por 54 ítems distribuidos en cinco áreas, y 21 competencias. Se utilizó dos escalas tipo Likert de seis puntos que valoran a) conocimiento y b) uso del docente respecto a las competencias digitales. Se consideran tres niveles: Básico, Intermedio y Avanzado. El llenado del instrumento fue realizado en línea. Los resultados son presentados mediante tablas de frecuencia relativa y correlación de Sperman.

\section{RESULTADOS}

Los resultados generales (Gráfico 1) muestran que la dimensión con mayor puntaje corresponde a Comunicación y colaboración, con 64\% de utilización y $67 \%$ de conocimiento. Esta área se encuentra en el nivel intermedio. La dimensión con el menor puntaje es Creación de contenido digital con 53\% de uso y 59\% de conocimiento, que también corresponde al nivel intermedio. Otro aspecto a destacar es que en todas las dimensiones, sobresale el conocimiento sobre la práctica con un promedio de 6 puntos, siendo el de mayor diferencia la dimensión Creación de contenido digital y la menor, Comunicación y colaboración, con 7 y 3 de diferencia respectivamente. En promedio, el nivel alcanzado en las cinco áreas es $63 \%$ en conocimiento y $58 \%$ en uso lo que corresponde a un nivel Intermedio de desarrollo de las competencias.

\section{Gráfico 1}

1. Información y alfabetización informacional

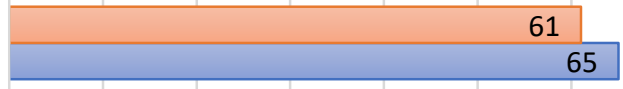

2. Comunicación y colaboración

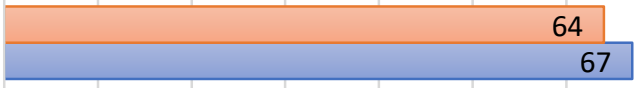

3. Creación de contenido digital

53 59

4. Seguridad

5. Resolución de problemas

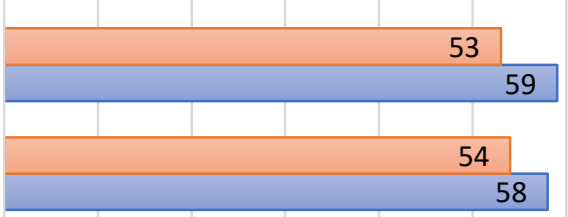

59

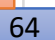

$\begin{array}{llllllll}0 & 10 & 20 & 30 & 40 & 50 & 60 & 70\end{array}$

80

$\square$ Utilizo $\square$ Conozco 


\section{Área 1: Información y alfabetización informacional}

Los resultados en primer lugar, destacan que hay una diferencia entre el nivel de conocimiento y el uso que se realiza (Tabla 3 ). El indicador más conocido y utilizado está referido a Navegación, búsqueda y filtrado, con $69 \%$ de conocimiento y $67 \%$ de uso. Esto puede deberse a la facilidad que brindan los navegadores. El indicador con menor porcentaje alcanzado es Evaluación de la información con $62 \%$ y $57 \%$ de conocimiento y uso, respectivamente. Este hecho es procupante puesto que, al haber bastante información el Internet, esta competencia debe tener un alto grado de desarrollo. Con relación al tercer indicador, se percibe que la población pierde información debido a que no realiza una adecuada gestión de conservación de los archivos digitales. Esto presupone un trabajo adicional cuando se busca nuevamente la información utilizada con anterioridad. En la dimensión Información y alfabetización informacional, la población estudiada tiene un $65 \%$ de conocimiento y $61 \%$ de uso, lo que la sitúa en un nivel Intermedio. Para alcanzar una competencia óptima (100\%) resta un 39\% de uso y $35 \%$ de conocimiento.

Tabla 3. Información y alfabetización informacional

\begin{tabular}{l|cc}
\hline \multicolumn{1}{c}{ Competencia } & Conozco & Utilizo \\
\hline 1.1 Navegación, busqueda y filtrado & $69 \%$ & $67 \%$ \\
1.2 Evaluación de información & $62 \%$ & $57 \%$ \\
1.3 Almacenamiento y recuperación & $63 \%$ & $58 \%$ \\
\hline
\end{tabular}

\section{Área 2: Comunicación y colaboración}

Esta dimensión permite la interacción con otras personas a través del uso de dispositivos y aplicaciones digitales. Hacer un uso adecuado de la comunicación en medios digitales y adaptarse a los diversos requerimientos de otros grupos. Los resultados en esta dimensión (Tabla 4) muestran que el más desarrollado es Participación en línea con 77\% de conocimiento y $74 \%$ de utilización. En Internet existen varios sitios en los cuales se puede participar, especialmente en redes sociales. Esto explicaría el nivel alcanzado en este indicador. El menos desarrollado es Gestión de identidad digital con 55\% y 50\% de conocimiento y uso. Este indicador muestra que aunque participa en línea, no lo hace con una adecuada gestión de su identidad digital. Si bien, muestran un buen desempeño de la competencia, aún requieren alcanzar un $33 \%$ en cuanto al conocimiento y $36 \%$ respecto al uso. Por tanto, se puede situar en un nivel Intermedio de desarrollo. 
Tabla 4. Comunicación y colaboración

\begin{tabular}{l|cc}
\hline \multicolumn{1}{c}{ Competencia } & Conozco & Utilizo \\
\hline 2.1 Interacción con tecnologías digitales & $71 \%$ & $68 \%$ \\
2.2 Compartir información y contenidos & $70 \%$ & $69 \%$ \\
2.3 Participación en línea & $77 \%$ & $74 \%$ \\
2.4 Colaboración mediante canales digitales & $65 \%$ & $55 \%$ \\
2.5 Netiqueta & $64 \%$ & $63 \%$ \\
2.6 Gestión de identidad digital & $55 \%$ & $50 \%$ \\
\hline
\end{tabular}

\section{Área 3: Creación de contenido digital}

Esta competencia incluye crear contenidos digitales en variedad de formatos. Asi, también, editar, mejorar y adaptar contenido ya existente a necesidades educativas. El indicador que obtuvo un porcentaje más alto es Desarrollo de contenidos digitales con $64 \%$ de conocimiento y $58 \%$ de uso (Tabla 5). El más bajo es el referido a derechos de autor y licencias con $50 \%$ y $44 \%$ de conocimiento y uso respectivamente. Esto debido a que en el contexto nacional no se practica de modo general, el respeto por derechos de autor y licencias y se utiliza de modo indiscrimindado material sin la debida autorización. Para alcanzar un desempeño óptimo en esta dimensión, se debe mejorar en un $41 \%$ respecto al conocimiento y $47 \%$ en el uso. El desarrollo de la competenciase sitúa en un nivel Intermedio.

Tabla 5. Creación de contenido digital

\begin{tabular}{l|cc}
\hline \multicolumn{1}{c}{ Competencia } & Conozco & Utilizo \\
\hline 3.1 Desarrollo de contenidos digitales & $64 \%$ & $58 \%$ \\
3.2 Integracíon y reelaboración & $57 \%$ & $50 \%$ \\
3.3 Derechos de autor y licencias & $50 \%$ & $44 \%$ \\
3.4 Programación & $52 \%$ & $49 \%$ \\
\hline
\end{tabular}

\section{Área 4: Seguridad}

Esta competencia incluye protección, tanto de dispositivos y contenidos digitales mediante el uso de medidas de seguridad de los mismos. El indicador más alto es Protección de salud y bienestar con $60 \%$ de conocimiento y $56 \%$ de uso (Tabla 6). Este indicador está relacionado con el cuidado de la integridad física y el bienestar psicológico en el uso de material digital y la navegación en Internet. El indicador con menor 
porcentaje es Protección del entorno, referido a optimizar los dispositivos de modo que se genere un mínimo impacto que afecte al medio ambiente. Para alcanzar un nivel óptimo, se requiere incrementar en un $42 \%$ en cuanto al conocimiento y en $46 \%$ respecto al uso.

Tabla 6. Seguridad

\begin{tabular}{l|cc}
\hline \multicolumn{1}{c}{ Competencia } & Conozco & Utilizo \\
\hline 4.1 Protección de dispositivos & $56 \%$ & $56 \%$ \\
4.2 Protección de datos personales & $59 \%$ & $57 \%$ \\
4.3 Protección de salud y bienestar & $60 \%$ & $56 \%$ \\
4.4 Protección del entorno & $53 \%$ & $46 \%$ \\
\hline
\end{tabular}

\section{Área 5: Resolución de problemas}

Esta competencia pretende resolver problemas técnicos básicos y luego, avanzados, relacionados con dispositivos y entornos digitales. El indicador en que se obtuvo el mayor porcentaje es Necesidades y respuestas tecnológicas, con $66 \%$ y $61 \%$ de conocimiento y uso. El indicador con menor porcentaje alcanzado es Resolución de problemas técnicos. En este indicador, el porcentaje alcanzado fue $61 \%$ en cuanto al conocimiento y $59 \%$ relacionado al uso (Tabla 7). De modo general, para que la competencia sea óptima, se debe incrementar un $46 \%$ en el conocimiento y $41 \%$ en el uso, lo que sitúa al desarrollo de la competencia en un nivel Intermedio.

Tabla 7. Resolución de problemas

\begin{tabular}{l|cc}
\hline \multicolumn{1}{c}{ Competencia } & Conozco & Utilizo \\
\hline 5.1 Resolución de problemas técnicos & $61 \%$ & $59 \%$ \\
5.2 Necesidades y respuestas tecnológicas & $66 \%$ & $61 \%$ \\
5.3 Innovación y uso de tecnología digital creativa & $63 \%$ & $58 \%$ \\
5.4 Lagunas en la competencia digital & $66 \%$ & $60 \%$ \\
\hline
\end{tabular}

\section{Correlación y prueba de hipótesis}

El estudio planteó las siguientes hipótesis referidas a la relación entre el nivel de conocimiento y el nivel de uso de las competencias digitales.

- $\mathrm{H}_{0}$ : No existe relación entre conocimiento y uso de competencias digitales

- $\mathrm{H}_{1}$ : Existe relación entre conocimiento y uso de competencias digitales 
En la Tabla 7 se puede establecer que el nivel de significancia (0.000) es menor a 0.01, por lo que se rechaza la hipótesis nula, entonces: existe relación lineal positiva de 0.992 entre el conocimiento desarrollado por los docentes y el uso que realiza el docente en el aula. Además, la correlación es alta y directamente proporcional.

Tabla 8. Coeficiente de correlación

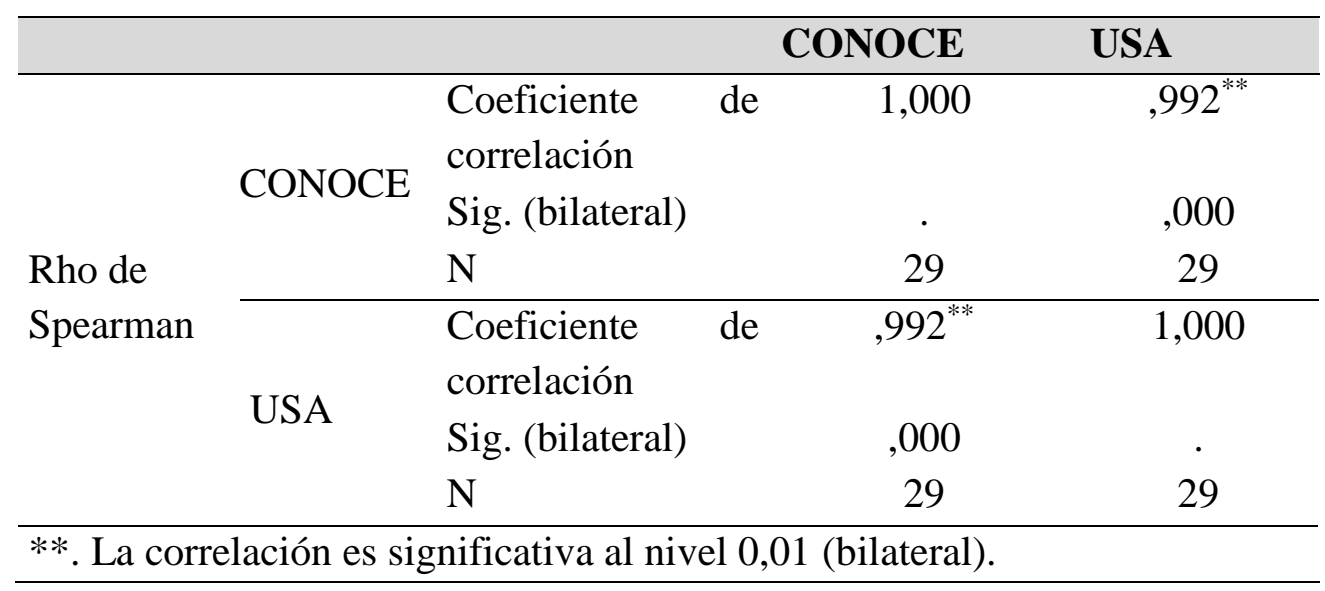

\section{DISCUSIÓN}

Información y alfabetización informacional. Los docentes localizan información y recursos digitales de su interés, aplicadas a la docencia universitaria. Expresan organizadamente sus necesidades en cuanto a información se refiere, la seleccionan y evalúan de acuerdo con criterios educativos. También son capaces de guardar, nombrar y recuperar archivos digitales utilizando estrategias de almacenamiento adecuados. Aunque muestran un nivel aceptable en esta dimensión, aún se debe trabajar bastante para el desarrollo adecuado de la competencia.

Comunicación y colaboración. Los participantes desarrollan procesos comunicativos haciendo una selección y uso de aplicaciones y servicios de comunicación digital adecuado a las necesidades educativas. Son capaces de interactuar sincrónica y asincrónicamente con los participantes de determinado curso. Participan activamente en redes sociales y educativas. Elaboran productos educativos no muy complejos para interactuar con los estudiantes utilizando medios digitales. En su interacción hacen uso de normas de netiqeta y respeta la divesidad cultural.

Creación de contenidos digitales. Los resultados muestran que la población de estudio es capaz de producir contenidos digitales en diferentes formatos: documentos de texto, infografías, presentaciones, diseño de imágenes, edición de vídeo o audio. Así también 
es promotor de los mismos en la comunidad universitaria. Conoce y utiliza repositorios de material bibliográrfico en línea. Puede modificar algunas aplicaciones para ajustarlas a sus necesidades. Diferencia entre licencias de uso abierto y cerrado.

Seguridad. La población estudiada es capaz de utilizar y gestionar información actualizada acerca de los peligros que conlleva el uso de dispositivos y contenido digital en la vida personal, profesional y educativa, así como el cuidado ergonómico y la adicción a las tecnologías. Tiene nociones elementales de recolección y uso de datos personales que realizan ciertas aplicaciones o sitios en la red.

Resolución de problemas. Desarrollaron habilidades para la evaluación y selección de servicios que ofrecen tanto los dispositivos como las aplicaciones con el fin de resolver dificultades y brindar soluciones a necesidades educativas. Se entiende que pueden resolver problemas técnicos no complejos tanto de dispositivos y aplicaciones con la ayuda de un manual o determinada información técnica disponible. Puede adaptar y complementa con otros, los medios digitales que la institución de proporcion, para ello, procura mantenerse actualizado con la finalidad de cubrir algunas necesidades que surjan en su labor pedagógica.

Respecto a la relación entre el conocimiento y uso de las competencias digitales, la correlación es alta y directamente proporcional. Los docentes que señalan un mayor conocimiento de las competencias informaciones digitales también indican un mayor uso. Aunque también puede verse que, en algunos casos, no siempre es así y que un mayor conocimiento no implica un mayor uso. Un estudio correlacional (Espino, 2018) encontró un nivel de significancia de 0,05 entre el desarrollo de competencias digitales de docentes y el desempeño pedagógico. Por su parte, Lamacponca-Román (2018) sostiene que existe una relación entre el entorno virtual de aprendizaje y las competencias digitales.

Los resultados del estudio hallan similitud con Rangel y Peñaloza (2013) en el sentido de que el nivel de conocimiento es más elevado que el de uso. A su vez, concuerda con Álvarez, Nuñez y Rodríguez (2017) en dos de las competencias con menos desarrollo como son Seguridad y Resolución de problemas. Si bien dicho estudio fue realizado en estudiantes universitarios, se observa el mismo panorama en los docentes. Se hallan coincidencias respecto a las dos competencias más desarrolladas Información y alfabetización informacional, Comunicación y colaboración con el estudio de Martínez y Garcés (2020) además, esta investigación también establece una relación positiva y fuerte 
en tres de las competencias, el presente estudio estableció una correlación alta y directamente proporcional. Por otra parte, los resultados contrastan con el estudio en el cual los docentes del nivel escolar tienen un nivel bajo en cuanto a sus competencias digitales (Zúñiga, 2020)

Los resultados encontrados sugieren acortar el nivel entre el conocimiento y uso, así se tendrían docentes con un mejor desempeño. Orientar la capacitación docente al desarrollo de las competencias digitales de tal modo que se alcance un nivel óptimo para pasar del nivel Intermedio, al Avanzado. En tal sentido, se podría contar con un equipo de docentes con la capacidad de generar y desarrollar procesos de aprendizaje mediados por entornos digitales (Aguilar \& Otuyemi. 2020; Amaya, Zúñiga, Salazar, Ávila, 2018). Esta labor comprende también fomentar en los estudiantes el desarrollo de competencias digitales y habilidades tecnológicas (Oliva \& Luna 2014; Sánchez 2021), hecho que repercutirá significativamente en la formación profesional.

\section{BIBLIOGRAFÍA}

Aguilar, R.; Otuyemi, E. (2020) La competencia digital es una necesidad permenente. Instituto para el futuro de la educación. Tecnológico de Monterrey. Recuperado de https://observatorio.tec.mx/edu-bits-blog/competencia-digital-una-necesidadpermanente

Álvarez-Flores, E.; Núñez-Gómez, P.; Rodríguez Crespo, C. (2017) Adquisición y carencia académica de competencias tecnológicas ante una economía digital. Revista Latina de Comunicación Social, 72, pp. 540-559.

Amaya, A.; Zúñiga, E.; Salazar, B.; Ávila, A. (2018). Empoderar a los profesores en su quehacer académico a través de certificaciones internacionales en competencias digitales. Apertura 10, 104-115.

Espino Wuffarden, J. E. (2018). Competencias digitales de los docentes y desempeño pedagógico en el aula. Universidad San Martín de Porres. Recuperado de: https://repositorio.usmp.edu.pe/bitstream/handle/20.500.12727/4525/espino_wje . pdf? sequence $=1 \&$ isAllowed $=\mathrm{y}$

Fernandez, F.; Fernández J. (2016) Los docentes de la Generación Z y sus competencias digitales. Revista Comunicar. N46, v XXIV. 97-105.

Hernández, P.; Fernández, C.; Baptista, M. (2014) Metodología de la investigación. México, McGrawHill. 
INTEF (2017) Marco común de competencias digital-docente. Ministerio de Educación, Cultura y Deporte, España.

Ketil, B. (2019) Comprendiendo los aspectos culturales y sociales de las competencias digitales. Comunicar. Revista Científica de Educomunicación.Nº 61, Vol. XXVII, 9-19.

Levano-Francia, L., Sanchez Diaz, S., Guillén-Aparicio, P., Tello-Cabello, S., HerreraPaico, N., \& Collantes-Inga, Z. (2019). Competencias digitales y educación. Propósitos y representaciones, 7(2), 569-588.

LLamacponca-Román, Y. (2018). Entornos virtuales de aprendizaje y desarrollo de competencias digitales en los docentes. Revista Científico Cultural, 7(1), 6.

Martínez-Garcés; J.; Garcés Fuentemayor, J. (2020) Competencias digitales y el reto de la educación virtual derivado de la covid-19. Educación y humanismo. 22 (39): pp. 1-16.

Navarro, E.; Barrios, S. (2016) Las competencias digitales en la educación superior. Revista de Transformación Educativa. Nº 1, 158-189.

Oliva, M. A., Coronas, T. T., \& Luna, J. C. Y. (2014). El desarrollo de competencias digitales en la educación superior. Historia y comunicación social, 19, 355-366.

Rangel, A.; Peñaloza, E. A. (2013) Alfbetización digital en docentes de educación superior: construcción y prueba empírica de un instrumento de evaluación. PixelBit. Revista de medios y educación. No 43, Julio, 9-23.

Sánchez Olavarría, C., \& Carrasco Lozano, M. E. E. (2021). COMPETENCIAS DIGITALES EN EDUCACIÓN SUPERIOR.Etic@net. Revista Científica Electrónica de Educación y Comunicación En La Sociedad Del Conocimiento, 21(1), 28-50.

Tourón, J., Martín, D., Navarro, E., Pradas, S. y Íñigo, V. (2018). Validación de constructo de un instrumento para medir la competencia digital docente de los profesores (CDD). Revista Española de Pedagogía, 76 (269), 25-54.

UNESCO, (2018) Las competencias digitales son esenciales para el empleo y la inclusión social. $\quad$ https://es.unesco.org/news/competencias-digitales-son-esencialesempleo-y-inclusion-social 
Vargas-Murillo, G. (2019). Competencias digitales y su integración con herramientas tecnológicas en educación superior. Cuadernos Hospital de Clínicas, 60(1), 8894.

Veiga de Cabo, Jorge, Fuente Díez, Elena de la, \& Zimmermann Verdejo, Marta. (2008). Modelos de estudios en investigación aplicada: conceptos y criterios para el diseño. Medicina y Seguridad del Trabajo, 54(210).

Zúñiga Reyes, J. F. (2020). Competencias digitales en docentes de una institución educativa, San Camilo, 2019. Repositorio Institucional - UCV. 